Published in final edited form as:

Curr Psychiatry Rep. ; 19(11): 84. doi:10.1007/s11920-017-0842-2.

\title{
Genetics of Anorexia Nervosa
}

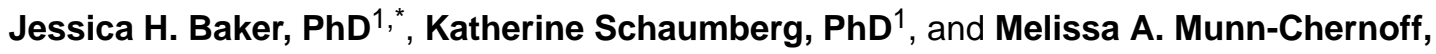
PhD ${ }^{1}$

${ }^{1}$ Department of Psychiatry, University of North Carolina, Chapel Hill, NC, USA

\section{Abstract}

Purpose of review: Genetic factors contribute to the etiology of anorexia nervosa (AN). This review synthesizes the current state of knowledge about the genetic etiology of AN, provides directions for future research, and discusses clinical implications for this research.

Recent findings: Candidate gene meta-analyses indicate serotonin genes may be involved in the genetic etiology of AN. Three genome-wide association studies have been conducted and one genome-wide significant locus was identified. Cross-disorder analyses suggest shared genetic risk between AN and several psychiatric, educational, and medical phenotypes.

Summary: Much has been learned about the genetic etiology of AN over the past three decades. However, to fully understand the genetic architecture, we must consider all aspects including common variation, cross-disorder analysis, rare variation, copy number variation, and geneenvironment interplay. Findings have important implications for the development of treatment and prevention approaches and for how AN, and psychiatric and medical diseases in general, are conceptualized.

\section{Keywords}

anorexia nervosa; psychiatry; genome-wide association studies; GWAS; 5-HT2A receptor gene; 5$H T T$ transporter gene

\section{Introduction}

According to the Diagnostic and Statistical Manual of Mental Disorders (DSM-5), anorexia nervosa (AN) is characterized by a body weight that is less then minimally expected for age, sex, developmental trajectory, and physical health, an intense fear of gaining weight, and body image disturbance [1]. The lifetime prevalence of DSM-5 AN is approximately 4\% [2], and AN is more common in females compared with males [3]. AN has the highest mortality rate of any psychiatric disorder [4] and is associated with high rates of medical complications and psychiatric comorbidities [3]. Despite the devastating consequences of AN, the etiology is still unclear. However, over the past three decades, research has established that genetic factors contribute to the etiology of AN. The aim of this review is to provide readers a brief overview of the history of genetic research as it pertains to AN, the

\footnotetext{
*Correspondence to: Dr. Baker, Department of Psychiatry, University of North Carolina at Chapel Hill, CB \#7160, 101 Manning Drive, Chapel Hill, NC 27599-7160, Voice: 984-974-3794 Fax: 984-974-3780, jhbaker@ med.unc.edu.

Human and Animal Rights. This article does not contain any studies with human or animal subjects performed by any of the authors.
} 
current state of knowledge about the genetic etiology of $\mathrm{AN}$, and the implications of this research. An exhaustive review of all genetic studies is not our aim; these have been reported elsewhere [5-7]. Here, we focus on historical studies and areas of research that have added to our knowledge about the genetic architecture of AN along with reports using current genetic methodologies to provide the reader with an accurate portrayal of our contemporary understanding of the genetic etiology of AN.

\section{Historical Genetic Findings}

Family and twin studies were among the first lines of evidence demonstrating genetic risk for AN (see Table 1 for definitions of genetic techniques). Family studies indicated that AN aggregates in families [8], and twin studies confirmed and extended findings related to the familial aggregation of AN, indicating there is a significant genetic contribution to this familial association. Replicated heritability estimates for AN have ranged between $48 \%$ $-74 \%$ depending on the definition of AN used [*5]. Twin studies have also suggested there is shared genetic risk between AN and multiple psychiatric phenotypes including other eating disorders [9], obsessive compulsive disorder [10], major depression, and suicide attempts [11]. Although family and twin studies provided initial evidence of the role of genetic factors in $\mathrm{AN}$, these methods cannot identify which genetic variants are involved in risk.

Early molecular genetic techniques used to identify genes involved in risk for AN included linkage studies and candidate gene studies (Table 1). Unfortunately, these methodologies revealed very little about precise genetic contributions to AN. Too few linkage studies were conducted and too few follow-ups were completed to draw definitive conclusions about the meaning of their findings. In contrast, hundreds of AN candidate gene studies have been conducted [5-7]; however, findings are hindered by small sample sizes, low power, and lack of replication. Thus, few conclusions can be drawn about the genes involved in risk for AN based on candidate gene studies. In the last decade, meta-analyses were conducted in an effort to clarify the fragmented candidate gene literature for the most promising single nucleotide polymorphisms (SNPs; variation at a single position in a DNA sequence among individuals). This included a serotonin receptor and transporter gene (HTR2A, 5-HTT), a $C O M T$ polymorphism (Val158Met), and a $B D N F$ polymorphism (Val66Met).

Meta-analysis results indicated a significant association between $H T R 2 A$ and AN. Specifically, the A allele of the $-1438 \mathrm{G} / \mathrm{A}$ polymorphism was significantly more common in individuals with AN compared with controls $[12,13]$. Two meta-analyses also indicated that the short allele of 5-HTTLPR, a 5-HTT polymorphism, increased risk for AN compared with long allele carriers [14, 15]. However, a meta-analysis conducted in 2016 did not replicate these results [*16]. Although some candidate gene studies showed a significant association between Val158Met and Val66Met and AN, meta-analyses indicated no significant association between these polymorphisms and AN risk [17, 18].

Undoubtedly, family, twin, linkage, and candidate gene studies paved the way for understanding the genetic architecture of AN. Several lessons were also learned from the inconsistent and inconclusive results obtained from linkage and candidate gene studies. This 
includes the limitations of a small sample size, heterogeneous methodologies, failure to account for multiple testing, and that a small set of candidate genes is not a valid model to explain the etiology of AN. Like other psychiatric disorders, AN is polygenic, whereby many genes of small effect contribute to its development. Thus, linkage and candidate gene studies are now considered out-of-date methodologies to identify genes involved in disease risk. Due to recent advances in technology and the decreased cost of genomic experiments, the psychiatric genetics field has moved on to more advanced methodologies such as genome-wide association studies (GWAS) and cross-disorder analysis (Table 1) that more closely align with a polygenic etiology. Unlike candidate gene studies, GWAS allow for millions of loci to be examined in a large number of individuals. Below, we review contemporary genetic findings in regard to the genetic architecture of AN. We focus this review on the most rigorously conducted, cutting-edge experiments during the past five years. Thus, pilot studies and/or studies small sample sizes are not reviewed, unless results are replicated, as definitive conclusions about findings cannot be made.

\section{Contemporary Genetic Findings}

\section{Genome-wide Association Studies}

Currently, three GWAS of AN have been published [19-21] (Table 2), but additional GWAS are underway [22]. The first two GWAS of AN conducted did not find any genome-wide significant results $[19,20]$. However, Boraska and colleagues (2014) [*20] observed that intronic variants in SOX2OT and PPP3CA were suggestively associated with AN, which have been previously associated with Alzheimer's disease. Despite the lack of genome-wide significant results, a majority of the replication results were in the same direction as the discovery results—a finding unlikely to be due to chance [20]. A smaller GWAS of 184 cases with bipolar disorder and a comorbid eating disorder also found suggestive hits within SOX2OT[23].

The largest and most rigorous GWAS to date included approximately 4,000 AN cases and 11,000 controls [**21]. One locus on chromosome 12 (12q13.2), which included six genes (IKZF4, RPS26, ERBB3, PA2G4, RPL41, and ZC3H1O), reached genome-wide significance $\left(p<5 \times 10^{-8}\right)$ and had a genome-wide common variant heritability of $20 \%$. The top SNP was in the ERBB3 gene. This SNP is in high linkage disequilibrium (i.e., non-random association of alleles at different loci) with a SNP associated with rheumatoid arthritis and type 1 diabetes. Previous associations with this region have also been reported with several other autoimmune diseases such as asthma, vitiligo, and alopecia areata. Interestingly, this GWAS did not confirm the previous suggestive associations observed between AN and SOX2OT or the results of candidate gene meta-analyses in regard to HTR2A and 5-HTT; thus, it is unknown whether these are potential true hits for AN or if GWAS to date have been underpowered to detect these effects.

\section{Cross-Disorder Analysis}

With the advent of GWAS and a growing repository of biological samples, it is now possible to examine shared genetic variants across disorders. These methodologies allow for identifying genetic overlap that occurs between multiple traits of interest. Although there are 
several novel methodologies developed to identify genetic overlap between disorders, only two have been applied to AN to date: linkage disequilibrium score regression (LDSC) and cross-trait analysis. LDSC is a statistical method used to determine the genetic correlation $\left(r_{g}\right)$ between phenotypes [**24]. LDSC uses GWAS summary statistics from case and control samples, accounts for linkage disequilibrium, and is not biased by sample overlap. Cross-trait analysis simply compares whether the genetic variants in a GWAS for one phenotype (e.g., AN) were found to be associated with a second phenotype, in an independent GWAS, based on a pre-determined significance or hit threshold (e.g., the top 1,000 most significant SNPs).

LDSC has been applied to examine the genetic relationship between AN and several other phenotypes. The first study, which had a relatively small GWAS AN sample available ( $\mathrm{n}=$ 2,907), observed a positive genetic correlation between AN and schizophrenia and a negative genetic correlation between AN and body mass index (BMI) [**24]. This suggests that the variants associated with schizophrenia increase risk for AN, whereas the variants associated with increased BMI decrease risk for AN. In contrast, a cross-trait analysis of risk alleles for AN and BMI showed that nine SNPs involved in risk for AN may also be associated with increased BMI [**25]. These findings suggest that the shared genetic risk for $\mathrm{AN}$ and $\mathrm{BMI}$ is complex and may involve some loci that predispose body weight in a directional manner (e.g. loci associated with propensity for lower body weight) along with others that do not function in a directional manner but may either increase or decrease body weight based on the influence of additional factors, such as the environment, as Hinney et al. suggests [**25]. Replication and further exploration of shared genetic risk for AN and BMI will shed light on the degree to which specific loci indicate directional risk or susceptibility to environmental influence on body weight.

In 2017, the largest LDSC study of AN was completed to date, which included 159 different phenotypes [21]. Findings indicated positive genetic correlations with neuroticism, schizophrenia, educational attainment, cross-disorder psychiatric risk, and HDL cholesterol, suggesting that variants associated with these phenotypes also increase risk for AN. Negative genetic correlations with obesity, normal range BMI, and other metabolic markers (e.g., insulin resistance, fasting glucose) were also observed, indicating that variants associated with increased risk for these phenotypes also decrease risk for AN. The negative correlations between AN and insulin resistance and AN and fasting insulin were the strongest genetic correlations among all the phenotypes examined, estimated at $r_{g}=-.50$ and $r_{g}=-.41$, respectively [21]. Further, the results of this study corroborate the genetic correlations in regard to AN and BMI discussed above indicating that the genetic risk for increased BMI is negatively associated with AN. The association observed between normal range BMI and AN also suggests that extremes of body weight (i.e., AN) are not necessarily regulated by genetic factors exclusive from normal variation in body weight. Going forward, examining cross-disorder associations may move beyond full-syndrome AN to explore continuous phenotypes and AN symptomatology with other psychiatric and medical phenotypes. 


\section{Future Perspectives}

Family and twin studies have demonstrated a significant genetic component for AN, and more contemporary molecular methods such as GWAS have begun to identify regions of the genome and variants that contribute to AN. Still, additional methods are available that will provide new avenues for identifying genetic markers for risk including the polygenic risk score (PRS), high-throughput sequencing, and copy number variation (CNV) (Table 1). Integrating this newfound genetic information-using multiple genomic approaches-with environmental risk factors will be key to providing comprehensive multifactorial risk prediction models. However, none of this will be possible without collaboration among researchers.

\section{Polygenic Risk Score}

The PRS [*26] uses GWAS information from all SNPs to create an individual genetic risk profile for association with a disorder. Varying $p$-value thresholds are examined for significance of a PRS and association with a disease in a discovery sample. The PRS derived in the discovery sample is then applied to an independent, target sample to examine the extent to which that PRS is associated with the phenotype. One advantage of this approach is that no genome-wide significance is necessary for SNPs to be included in the analyses-the analysis is on SNPs that may not necessarily meet the strict $p$-value threshold, thus maximizing the amount of genomic data already available. Individuals from the target sample are divided into groups (e.g., quartiles) based on their PRS to examine whether individuals who are at highest genetic risk are more likely to have AN compared with individuals at lowest genetic risk. A PRS for AN is under development.

Although there are no published studies for AN using this method, it has been informative for other psychiatric disorders. For example, the PRS for schizophrenia explains nearly $18 \%$ of the genetic variation in that disorder [27]. Additional benefits of the PRS are that environmental variables can easily be included in the model (see below) and can be applied for use in other psychiatric disorders or related traits such that it is another method to examine cross-disorder associations. Specifically, PRS of AN can be applied to other psychiatric phenotypes and medical or physical traits to evaluate shared risk (and vice versa). Although the PRS has the potential to provide powerful information on genetic etiology, caution is critical when interpreting any findings. Not only will increasing sample sizes improve prediction models [27], but it is essential that this information be coupled with additional genomic and environmental information to create more accurate multimodal risk prediction models.

\section{High-throughput Sequencing}

Researchers have long debated whether common variants of small effect (termed "common disease, common variant" hypothesis) or rare variants of large effect (termed "common disease, rare variant" hypothesis) influence risk for psychiatric disorders. Common variants are genetic variants where the minor allele occurs in more than $5 \%$ of the population, whereas rare variants describe genetic variants in which the minor allele is present in less than $5 \%$ of the population. The genomic methods discussed herein have focused exclusively 
on common variants because they are easier to detect since they are present in a vast majority of individuals. On the other hand, rare variants may have higher disease penetrance, but are only present in a small number of individuals. This makes detection difficult as sample sizes on par with what is needed for GWAS are necessary. Ultimately, the findings from studies examining both hypotheses will provide the greatest insight into genetic risk for AN.

High-throughput DNA sequencing is a popular method used to identify rare variants. Two common high-throughput DNA sequencing methods are exome sequencing, which sequences exons on all genes across the genome, and whole-genome sequencing, which provides an individual's entire DNA sequence. Although examining rare variants will likely yield important insight into genetic risk for AN for some individuals, this is a new direction for the eating disorder field and only three preliminary studies of AN have been published to date. The first study used a family pedigree design, which observed two mutations to segregate with AN [28] and the second a small candidate gene study design $(\mathrm{n}=\sim 3,000)$ observing associations between AN and two genes [29]. Although these findings show promise, studies with much larger sample sizes are necessary. The third and most recent high-throughput sequencing study of AN did not find associations between AN and common, low frequency variants or rare variants [30]. Nevertheless, integrating information from both common and rare variants will strengthen our understanding of genetic risk for AN.

\section{Copy Number Variation}

$\mathrm{CNV}$ is another type of genome-wide study to examine genetic risk for disease. CNVs occur when sections of the genome are repeated or deleted and this repetition or deletion varies between individuals. In 2017, the largest CNV survey of AN was conducted [*31]. This report included approximately 2,000 AN cases and examined the prevalence of rare CNVs previously associated with psychiatric and neurodevelopmental disorders. Two cases had CNVs previously associated with disease-a deletion region associated with autism, schizophrenia, and developmental delay and a deletion region previously associated with AN in a pilot study [32]. Additionally, 40 other regions containing large, rare CNVs were identified, many of which showed no previous associations with a psychiatric or neurodevelopmental disorder.

\section{Gene and Environment Interplay}

Genes and environment work jointly in the development of AN via two ways: (1) geneenvironment correlation (rGE) and (2) gene-by-environment interaction (GxE) (Table 1). rGE occurs when environmental factors influence genetic risk for a trait. For example, an individual who has a genetic predisposition for AN may participate in weight-focused sports (e.g., gymnastics, wrestling) and/or may associate with peer groups who stress the importance of a particular body weight and shape. Unfortunately, no rGE studies in AN have been conducted.

Conversely, GxE occurs when environmental factors enhance or reduce genetic risk or when genetic risk influences response to an environment. It is more frequently discussed than rGE 
in AN, perhaps because it is easier to investigate in molecular genetic approaches. Modern genomic approaches are beginning to integrate specific environmental risks (e.g., childhood trauma) to determine whether having a particular genetic variant increases risk for AN in the presence of experiencing a significant life event. For example, in one small GxE study, Karwautz et al. (2011) [*33] reported that women with AN who had at least one short allele of 5-HTTLPR reported more problematic parenting styles growing up than their biological sisters who did not have AN. Although limited in scope because of the large sample sizes needed for genomic studies, studies similar to this one will undoubtedly lead to new scientific discoveries.

\section{Collaborations and Consortiums}

One lesson learned from the history of psychiatric genetics and the increasing popularity of GWAS is the need for very large sample sizes. It is now generally accepted that a minimum sample size of 10,000 cases and 10,000 controls may be necessary to find sufficient significant hits in GWAS for polygenic disease. This means the psychiatric genetics field needs to become a field of collaboration. The sample sizes necessary to find meaningful results are next to impossible for a single scientific researcher to obtain. As such, in 2007, the Psychiatric Genomics Consortium (PGC) was established. The PGC includes over 800 researchers from 38 different countries and more than 900,000 individual samples. To date, the PGC has 13 working groups representing differing areas of interest in psychiatric genetics, including eating disorders, which joined the PGC approximately four years ago.

Unfortunately, the eating disorders field has historically lagged behind in genetics research. The AN field is beginning to make strides, but much work is to be done. Developing the PGC Eating Disorders Working Group is a start and making further advances will depend on continued collaboration. For example, we were fortunate to be part of the largest collaboration ever aimed at identifying genes involved in AN risk: the Anorexia Nervosa Genetics Initiative (ANGI). This was a worldwide collaboration in the United States (University of North Carolina), Australia, New Zealand, Sweden, and Denmark, and will represent the largest GWAS of AN ever conducted. The future of science depends on the willingness of investigators to collaborate. Meaningful science can no longer occur in a bubble.

\section{Clinical Implications}

Although an era in which individual-level genetic information can be used for clinical care of AN has not yet arrived, that is the ultimate goal of much of this work. Despite this, basic scientific understanding of the role of genetic risk in eating disorders can be integrated into clinical practice today. One of the most pertinent implications from the currently available research is understanding, and patients and families should be provided psychoeducation in regard to the genetic etiology of AN. For example, recently, widely disseminated resources for treatment providers and patients have included statements about the role of genetics in risk for eating disorders (e.g. Truths \#7 and 8 from the Academy for Eating Disorders' Nine Truths about Eating Disorders [34]). Moreover, it is a treatment provider's responsibility to 
provide patients with education about all of the factors that may have played a role in the development of the illness, including genetic factors.

Providing patients and caregivers with information regarding genetic and biological underpinnings as part of our understanding of AN does present some clinical issues. One study using semi-structured interviews found that patients, in general, anticipated that genetic reframing of eating disorder etiology would reduce eating disorder stigma, whereas half of patients surveyed also anticipated that genetic framing of the disease could hamper their recovery efforts [34]. Moving forward, development of accurate, digestible clinical resources to explain genetic risk to patients, including how such factors fit into a broad conceptualization of AN risk and recovery, is necessary. Genetic information can also play a valuable role in prevention. Although we are not there yet, the ability to identify genetic markers of risk could allow for early screening in those at high risk so that intervention and prevention efforts can be focused on those who are most vulnerable-stopping the disease before it starts.

Genetic correlations of AN with other traits and conditions suggest there may exist shared, overlapping genetic networks that regulate behavior. Further probing of these genetic correlations will be useful for understanding clinical approaches that might be best suited for a next generation of interventions. The use of animal models to determine genetic influences on feeding and metabolism, which is beyond the scope of this review, can improve understanding of genes that might represent novel, specific pharmacotherapeutic targets for AN. For example, pharmacological agents that address metabolic or autoimmune disease identified by GWAS and LDSC findings may represent an innovative avenue to pursue for treatment. In addition, clinical research can use PRS to examine treatment response, which will eventually provide assistance in individually tailoring treatment and prevention efforts [*36]. Findings can also be included in multifactorial risk estimation algorithms that account for other genetic factors (e.g., rare variants) and environmental risk factors to improve prediction.

\section{Conclusion}

We have learned a lot about the genetic architecture of AN over the past three decades. However, in order to fully understand the genetic architecture of AN, we must consider all aspects including common variation, rare variation, CNVs, cross-disorder analysis, and gene environment interplay - all of which we know little about. Early molecular genetic techniques such as linkage and candidate gene studies have fallen out of favor for these more advanced methodologies. Despite this, meta-analyses attempting to synthesize the candidate gene literature indicate that serotonin receptor and transporter genes could be involved in the genetic etiology of AN; in particular, HTR2A and 5-HTT. However, these findings have not been replicated by more contemporary genomic methods.

The eating disorders field is starting to gain traction in modern molecular genetic techniques such as GWAS and cross-disorder analysis. The first genome-wide significant locus for risk for AN has been identified. This locus has been identified previously as involved in autoimmune disease and type 1 diabetes. Cross-disorder analyses have also suggested shared 
genetic risk between AN and several psychiatric, educational, and medical phenotypeswith the highest overlap observed between insulin resistance and fasting insulin. Identifying a genome-wide significant locus that associates with autoimmune disease coupled with these significant genetic correlations for metabolic indicators suggest that AN may be better conceptualized as a disorder that arises from both psychiatric and metabolic etiologies.

Indeed, results of cross-disorder analyses indicate that our current diagnostic classification system does not represent etiologically distinct disorders. The arbitrary distinctions made between psychiatric disorders themselves and between psychiatric disorders and other medical diseases (e.g., type 1 diabetes) do not account for the possibility of a shared genetic etiology. Specifically, the positive genetic correlation observed between AN and schizophrenia indicates a partially shared genetic etiology. Genetic correlations observed between AN and weight-related measures (e.g., obesity, BMI) also suggest that at least some of the genetic factors that influence normal variation in BMI and weight-dysregulation extremes (e.g., AN and obesity) are shared [24, 25]—-meaning that aspects of weight that were thought to have distinct etiologies in fact, overlap genetically. This overlap may involve some loci that influence weight in a directional manner (i.e. predisposing to either higher or lower body weight) and others that influence body weight based on the presence of additional factors. This hypothesis is consistent with models of psychopathology highlighting that, in some cases, genes may dispose "plasticity" of traits, rendering some individuals more malleable to environmental influences [37]. Moving forward, molecular genetic examinations of AN need to move beyond the full-syndrome to explore symptomlevel associations with other psychiatric and medical related traits in a cross-disorder manner. Results from such analyses can be formative in developing classification symptoms based on overlapping etiologies versus subjective distinctions. Finally, researchers must work with treatment providers to understand genetic findings so they can educate patients about the role of genes in the risk for AN and what this might mean for treatment and recovery. As the genetics field evolves, eventually genetic information will be directly used in the treatment for and prevention of AN.

\section{Acknowledgments}

Dr. Baker was supported by grant NIH K01MH106675; Dr. Munn-Chernoff was supported by grant NIH K01AA025113; Dr. Schaumberg was supported by NIH grant T32MH076694 (PI: Cynthia Bulik, PhD).

\section{References}

1. American Psychiatric Association Diagnostic and Statistical Manual of Mental Disorders 5th Edition. Arlington, VA: American Psychiatric Publishing; 2013.

2. Mustelin L, Silen Y, Raevuori A, Hoek HW, Kaprio J, Keski-Rahkonen A. The DSM-5 diagnostic criteria for anorexia nervosa may change its population prevalence and prognostic value. J Psychiatr Res 2016;77:85-91. 10.1016/j.jpsychires.2016.03.003. [PubMed: 27014849]

3. Hudson JI, Hiripi E, Pope HG, Jr, Kessler RC. The prevalence and correlates of eating disorders in the National Comorbidity Survey Replication. Biol Psychiatry 2007;61:348-58. 10.1016/j.biopsych. 2006.03.040. [PubMed: 16815322]

4. Chesney E, Goodwin GM, Fazel S. Risks of all-cuse and suicide mortality in mental disorders: a meta-review. World Psychiatry 2014;13:153-60. 10.1002/wps.20128 [PubMed: 24890068] 
5*. Trace SE, Baker JH, Penas-Lledo E, Bulik CM. The genetics of eating disorders. Annu Rev of Clin Psychol 2013;9:589-620. 10.1146/annurev-clinpsy-050212-185546. In depth review of all genetic studies conducted examining the genetic risk for eating disorders. [PubMed: 23537489]

6. Monteleone P, Maj M. Genetic susceptibility to eating disorders: associated polymorphisms and pharmacogenetic suggestions. Pharmacogenomics 2008;9:1487-520. 10.2217/14622416.9.10.1487. [PubMed: 18855537]

7. Hinney A, Volckmar AL. Genetics of eating disorders. Curr Psychiatry Rep 2013;15:423 10.1007/ s11920-013-0423-y. [PubMed: 24202964]

8. Strober M, Freeman R, Lampert C, Diamond J, Kaye W. Controlled family study of anorexia nervosa and bulimia nervosa: evidence of shared liability and transmission of partial syndromes. Am J Psychiatry 2000;157:393-401. 10.1176/appi.ajp.157.3.393 [PubMed: 10698815]

9. Bulik CM, Thornton LM, Root TL, Pisetsky EM, Lichtenstein P, Pedersen NL. Understanding the relation between anorexia nervosa and bulimia nervosa in a Swedish national twin sample. Biol Psychiatry 2010;67(1):71-7. 10.1016/j.biopsych.2009.08.010 [PubMed: 19828139]

10. Cederlof M, Thornton LM, Baker J, Lichtenstein P, Larsson H, Ruck C et al. Etiological overlap between obsessive-compulsive disorder and anorexia nervosa: a longitudinal cohort, multigenerational family and twin study. World Psychiatry 2015;14(3):333-8. 10.1002/wps.20251. [PubMed: 26407789]

11. Thornton LM, Welch E, Munn-Chernoff MA, Lichtenstein P, Bulik CM. Anorexia nervosa, major depression, and suicide attempts: shared genetic factors. Suicide Life Threat Behav 2016;46:52534. 10.1111/sltb.12235. [PubMed: 26916469]

12. Gorwood P, Kipman A, Foulon C. The human genetics of anorexia nervosa. Eur J Pharmacol 2003;480:163-70. [PubMed: 14623359]

13. Martaskova D, Slachtova L, Kemlink D, Zahorakova D, Papezova H. Polymorphisms in serotoninrelated genes in anorexia nervosa. The first study in Czech population and metaanalyses with previously performed studies. Folia Biol (Praha) 2009;55:192-7. [PubMed: 19863848]

14. Calati R, De Ronchi D, Bellini M, Serretti A. The 5-HTTLPR polymorphism and eating disorders: a meta-analysis. Int J Eat Disord 2011;44:191-9. 10.1002/eat.20811. [PubMed: 20209488]

15. Lee Y, Lin PY. Association between serotonin transporter gene polymorphism and eating disorders: a meta-analytic study. Int J Eat Disord 2010;43:498-504. 10.1002/eat.20732. [PubMed: 19708070]

16*. Solmi M, Gallicchio D, Collantoni E, Correll CU, Clementi M, Pinato C et al. Serotonin transporter gene polymorphism in eating disorders: Data from a new biobank and META-analysis of previous studies. World J Biol Psychiatry 2016;17:244-57. 10.3109/15622975.2015.1126675. Most recent meta-analysis of the association between 5-HTTLRP and risk for AN, showing no significant association. [PubMed: 26895183]

17. Brandys MK, Kas MJ, van Elburg AA, Ophoff R, Slof-Op't Landt MC, Middeldorp CM et al. The Val66Met polymorphism of the BDNF gene in anorexia nervosa: New data and a meta-analysis. World J Biol Psychiatry 2011;14:441-51. 10.3109/15622975.2011.605470. [PubMed: 21936709]

18. Brandys MK, Slof-Op't Landt MC, van Elburg AA, Ophoff R, Verduijn W, Meulenbelt I et al. Anorexia nervosa and the Val158Met polymorphism of the COMT gene: meta-analysis and new data. Psychiatr Genet 2012;22:130-6. 10.1097/YPG.0b013e328351859e. [PubMed: 22366815]

19. Wang K, Zhang H, Bloss CS, Duvvuri V, Kaye W, Schork NJ et al. A genome-wide association study on common SNPs and rare CNVs in anorexia nervosa. Mol Psychiatry 2011;16:949-59. 10.1038/mp.2010.107. [PubMed: 21079607]

20*. Boraska V, Franklin CS, Floyd JA, Thornton LM, Huckins LM, Southam L et al. A genome-wide association study of anorexia nervosa. Mol Psychiatry 2014;19:1085-94. 10.1038/mp.2013.187. First large-scale genome-wide association study of AN. [PubMed: 24514567]

21**. Duncan L, Yilmaz Z, Gaspar H, Walters R, Goldstein J, Anttila V et al. Significant locus and metabolic genetic correlations revealed in genome-wide association study of anorexia bervosa. Am J Psychiatry 10.1176/appi.ajp.2017.16121402. First genome-wide assosociation study of AN to find a significant genetic loci as well as significant genetic correlations between AN and metabolic markers.

Curr Psychiatry Rep. Author manuscript; available in PMC 2018 September 22. 
22. Kirk KM, Martin FC, Mao A, Parker R, Maguire S, Thornton LM et al. The Anorexia Nervosa Genetics Initiative: Study description and sample characteristics of the Australian and New Zealand arm. Aust N Z J Psychiatry 10.1177/0004867417700731.

23. Liu X, Bipolar Genome S, Kelsoe JR, Greenwood TA. A genome-wide association study of bipolar disorder with comorbid eating disorder replicates the SOX2-OT region. J Affect Disord 2016;189:141-9. 10.1016/j.jad.2015.09.029. [PubMed: 26433762]

24**. Bulik-Sullivan B, Finucane HK, Anttila V, Gusev A, Day FR, Loh PR et al. An atlas of genetic correlations across human diseases and traits. Nat Genet 2015;47:1236-41. 10.1038/ng.3406. First LD score regression study including AN, which showed significant genetic correlations between multiple medical, psychiatric, and educational phenotypes and AN. [PubMed: 26414676]

25**. Hinney A, Kesselmeier M, Jall S, Volckmar AL, Focker M, Antel J et al. Evidence for three genetic loci involved in both anorexia nervosa risk and variation of body mass index. Mol Psychiatry 2017;22:192-201. 10.1038/mp.2016.71. Cross-trait analysis of AN and BMI indicating genetic variants involved in risk for AN may also be associated with increased BMI. [PubMed: 27184124]

26*. Wray NR, Lee SH, Mehta D, Vinkhuyzen AA, Dudbridge F, Middeldorp CM. Research review: Polygenic methods and their application to psychiatric traits. J Child Psychol Psychiatry 2014;55:1068-87. 10.1111/jcpp.12295. Review of the potential implications of polygenic research methods. [PubMed: 25132410]

27. Schizophrenia Working Group of the Psychiatric Genomics C. Biological insights from 108 schizophrenia-associated genetic loci. Nature 2014;511:421-7. 10.1038/nature13595. [PubMed: 25056061]

28. Cui H, Moore J, Ashimi SS, Mason BL, Drawbridge JN, Han S et al. Eating disorder predisposition is associated with ESRRA and HDAC4 mutations. J Clin Invest 2013;123:4706-13. 10.1172/JCI71400. [PubMed: 24216484]

29. Scott-Van Zeeland AA, Bloss CS, Tewhey R, Bansal V, Torkamani A, Libiger O et al. Evidence for the role of EPHX2 gene variants in anorexia nervosa. Mol Psychiatry 2014;19:724-32. 10.1038/mp.2013.91. [PubMed: 23999524]

30. Huckins LM, Hatzikotoulas K., Southam L, Thornton L, Steinberg J, Aguilera-McKay F. et al. Investigation of common, low-frequency and rare genome-wide variation in anorexia nervosa. Mol Psychiatry in press.

31*. Yilmaz Z, Szatkiewicz JP, Crowley JJ, Ancalade N, Brandys MK, van Elburg A et al. Exploration of large, rare copy number variants associated with psychiatric and neurodevelopmental disorders in individuals with anorexia nervosa. Psychiatr Genet 10.1097/YPG.0000000000000172. Largest CNV study of AN conducted to date.

32. Wang K, Zhang H, Bloss CS, Duvvuri V, Kaye W, Schork NJ et al. A genome-wide association study on common SNPs and rare CNVs in anorexia nervosa. Mol Psychiatry 2011;16:949-59. 10.1038/mp.2010.107. [PubMed: 21079607]

33*. Karwautz AF, Wagner G, Waldherr K, Nader IW, Fernandez-Aranda F, Estivill X et al. Geneenvironment interaction in anorexia nervosa: relevance of non-shared environment and the serotonin transporter gene. Mol Psychiatry 2011;16:590-2. 10.1038/mp.2010.125. The only gene by environment interaction study of AN published to date. [PubMed: 21135854]

34. Academy for Eating Disorders. Nine Truths about Eating Disorders Retrieved August 21, 2017. https://www.aedweb.org/index.php/10-news/171-9-truths-about-eating-disorders

35. Easter MM. "Not all my fault": genetics, stigma, and personal responsibility for women with eating disorders. Soc Sci Med 2012;75:1408-16. 10.1016/j.socscimed.2012.05.042. [PubMed: 22819736]

36*. Chatterjee N, Shi J, Garcia-Closas M. Developing and evaluating polygenic risk prediction models for stratified disease prevention. Nat Rev Genet 2016;17:392-406. 10.1038/nrg.2016.27. Provides a summary of the methods used for risk prediction models that include genetic and nongenetic information and potential applications. [PubMed: 27140283]

37. Belsky J, Pluess M. Beyond diathesis stress: differential susceptibility to environmental influences. Psychol Bull 2009;135:885-908. 10.1037/a0017376. [PubMed: 19883141] 
Table 1.

Definitions of historical and contemporary molecular genetic techniques

\begin{tabular}{|c|c|}
\hline Method & Definition \\
\hline Family study & $\begin{array}{l}\text { Compares the prevalence of a trait of interest in family members of individuals with the trait of interest to family } \\
\text { members of those without the trait of interest. }\end{array}$ \\
\hline Twin study & $\begin{array}{l}\text { Correlations between monozygotic (MZ) and dizygotic (DZ) twins for a trait of interest are compared to } \\
\text { determine } \\
\text { the extent to which variation in the trait is due to genetic and environmental factors. Because MZ twins are } \\
\text { presumed to share } 100 \% \text { and DZ twins } 50 \% \text { of their genetic makeup, if the correlation for a trait is twice as } \\
\text { strong } \\
\text { in MZ than DZ twins, genetic factors are implicated. }\end{array}$ \\
\hline Linkage study & $\begin{array}{l}\text { Identifies regions of the genome involved in genetic risk for a trait through samples of multiplex families. Does } \\
\text { not } \\
\text { identify specific genes involved in risk, only chromosomal regions. }\end{array}$ \\
\hline Candidate gene study & $\begin{array}{l}\text { Examines the allele frequency of an a priori selected single nucleotide polymorphism (SNP) in individuals with } \\
\text { and without a trait of interest. }\end{array}$ \\
\hline $\begin{array}{l}\text { Genome-wide association } \\
\text { study (GWAS) }\end{array}$ & $\begin{array}{l}\text { Compares individuals with and without a trait of interest on } 300,000 \text { to } 1,000,000 \text { genetic markers across the } \\
\text { genome simultaneously. Genome-wide statistical significance is indicated by } p<5 \times 10^{-8} \text {. }\end{array}$ \\
\hline $\begin{array}{l}\text { Copy number variation } \\
(\mathrm{CNV})\end{array}$ & Examines the presence of genomic repeats or deletions in individuals with trait of interest. \\
\hline $\begin{array}{l}\text { Linkage disequilibrium } \\
\text { score regression (LDSC) }\end{array}$ & Statistical method used to determine the genetic correlation between traits. \\
\hline Polygenic risk score (PRS) & Uses GWAS information from all SNPs to create an individual genetic risk profile for association with a trait. \\
\hline High-throughput sequencing & Uses next-generation DNA sequencing techniques to examine variants associated with disease. \\
\hline $\begin{array}{l}\text { Gene-environment } \\
\text { correlation (rGE) }\end{array}$ & Environmental factors influence genetic risk for a trait. \\
\hline $\begin{array}{l}\text { Gene-by-environment } \\
\text { interaction }(\mathrm{GxE})\end{array}$ & $\begin{array}{l}\text { Environmental factors enhance or reduce genetic risk or when genetic risk influences response to an } \\
\text { environment. }\end{array}$ \\
\hline
\end{tabular}

Curr Psychiatry Rep. Author manuscript; available in PMC 2018 September 22. 
Table 2.

Currently published genome-wide association studies of anorexia nervosa

\begin{tabular}{llccl}
\hline \multicolumn{1}{c}{ Study } & Sample Size & $\begin{array}{c}\text { Genome-wide } \\
\text { Significant Finding }\end{array}$ & Top SNP(s) & Candidate Gene(s) \\
\hline $\begin{array}{l}\text { Wang et al., 2011 } \\
{[19]}\end{array}$ & $\begin{array}{l}1,033 \text { cases } \\
3,733 \text { controls }\end{array}$ & No & rs533123, rs7532266 & OPRD1, HTR1D \\
$\begin{array}{l}\text { Boraska et al., } \\
2014[20]\end{array}$ & $\begin{array}{l}2,907 \text { cases } \\
14,860 \text { controls }\end{array}$ & No & rs9839776, rs17030795 & SOX2OT, PPP3CA \\
$\begin{array}{l}\text { Duncan et al., in } \\
\text { press [21] }\end{array}$ & $\begin{array}{l}3,495 \text { cases } \\
10,982 \text { controls }\end{array}$ & Yes & rs4622308 & ERBB3 \\
\hline
\end{tabular}

$\mathrm{SNP}=$ single nucleotide polymorphism . 